\title{
Strong Convergence Theorems of the General Iterative Methods for Nonexpansive Semigroups in Banach Spaces
}

\author{
Rattanaporn Wangkeeree ${ }^{1,2}$ \\ ${ }^{1}$ Department of Mathematics, Faculty of Science, Naresuan University, Phitsanulok 65000, Thailand \\ ${ }^{2}$ Centre of Excellence in Mathematics, CHE, Si Ayutthaya Road, Bangkok 10400, Thailand
}

Correspondence should be addressed to Rattanaporn Wangkeeree, rattanapornw@nu.ac.th

Received 4 February 2011; Accepted 22 March 2011

Academic Editor: Yonghong Yao

Copyright (c) 2011 Rattanaporn Wangkeeree. This is an open access article distributed under the Creative Commons Attribution License, which permits unrestricted use, distribution, and reproduction in any medium, provided the original work is properly cited.

Let $E$ be a real reflexive Banach space which admits a weakly sequentially continuous duality mapping from $E$ to $E^{*}$. Let $\mathcal{S}=\{T(s): 0 \leq s<\infty\}$ be a nonexpansive semigroup on $E$ such that $\operatorname{Fix}(\mathcal{S}):=\bigcap_{t>0} \operatorname{Fix}(T(t)) \neq \emptyset$, and $f$ is a contraction on $E$ with coefficient $0<\alpha<1$. Let $F$ be $\delta$-strongly accretive and $\lambda$-strictly pseudocontractive with $\delta+\lambda>1$ and $\gamma$ a positive real number such that $\gamma<1 / \alpha(1-\sqrt{1-\delta / \lambda})$. When the sequences of real numbers $\left\{\alpha_{n}\right\}$ and $\left\{t_{n}\right\}$ satisfy some appropriate conditions, the three iterative processes given as follows: $x_{n+1}=$ $\alpha_{n} \gamma f\left(x_{n}\right)+\left(I-\alpha_{n} F\right) T\left(t_{n}\right) x_{n}, n \geq 0, y_{n+1}=\alpha_{n} \gamma f\left(T\left(t_{n}\right) y_{n}\right)+\left(I-\alpha_{n} F\right) T\left(t_{n}\right) y_{n}, n \geq 0$, and $z_{n+1}=T\left(t_{n}\right)\left(\alpha_{n} \gamma f\left(z_{n}\right)+\left(I-\alpha_{n} F\right) z_{n}\right), n \geq 0$ converge strongly to $\tilde{x}$, where $\tilde{x}$ is the unique solution in $\operatorname{Fix}(\mathcal{S})$ of the variational inequality $\langle(F-\gamma f) \tilde{x}, j(x-\tilde{x})\rangle \geq 0, x \in \operatorname{Fix}(\mathcal{S})$. Our results extend and improve corresponding ones of Li et al. (2009) Chen and He (2007), and many others.

\section{Introduction}

Let $E$ be a real Banach space. A mapping $T$ of $E$ into itself is said to be nonexpansive if $\| T x-$ $T y\|\leq\| x-y \|$ for each $x, y \in E$. We denote by $\operatorname{Fix}(T)$ the set of fixed points of $T$. A mapping $f: E \rightarrow E$ is called $\alpha$-contraction if there exists a constant $0<\alpha<1$ such that $\|f(x)-f(y)\| \leq$ $\alpha\|x-y\|$ for all $x, y \in E$. A family $\mathcal{S}=\{T(t): 0 \leq t<\infty\}$ of mappings of $E$ into itself is called a nonexpansive semigroup on $E$ if it satisfies the following conditions:

(i) $T(0) x=x$ for all $x \in E$;

(ii) $T(s+t)=T(s) T(t)$ for all $s, t \geq 0$; 
(iii) $\|T(t) x-T(t) y\| \leq\|x-y\|$ for all $x, y \in E$ and $t \geq 0$;

(iv) for all $x \in E$, the mapping $t \mapsto T(t) x$ is continuous.

We denote by $\operatorname{Fix}(\mathcal{S})$ the set of all common fixed points of $\mathcal{S}$, that is,

$$
\operatorname{Fix}(\mathcal{S}):=\{x \in E: T(t) x=x, 0 \leq t<\infty\}=\bigcap_{t \geq 0} \operatorname{Fix}(T(t))
$$

In [1], Shioji and Takahashi introduced the following implicit iteration in a Hilbert space

$$
x_{n}=\alpha_{n} x+\left(1-\alpha_{n}\right) \frac{1}{t_{n}} \int_{0}^{t_{n}} T(s) x_{n} d s, \quad \forall n \in \mathbb{N},
$$

where $\left\{\alpha_{n}\right\}$ is a sequence in $(0,1)$ and $\left\{t_{n}\right\}$ is a sequence of positive real numbers which diverges to $\infty$. Under certain restrictions on the sequence $\left\{\alpha_{n}\right\}$, Shioji and Takahashi [1] proved strong convergence of the sequence $\left\{x_{n}\right\}$ to a member of $F(\mathcal{S})$. In [2], Shimizu and Takahashi studied the strong convergence of the sequence $\left\{x_{n}\right\}$ defined by

$$
x_{n+1}=\alpha_{n} x+\left(1-\alpha_{n}\right) \frac{1}{t_{n}} \int_{0}^{t_{n}} T(s) x_{n} d s, \quad \forall n \in \mathbb{N}
$$

in a real Hilbert space where $\{T(t): t \geq 0\}$ is a strongly continuous semigroup of nonexpansive mappings on a closed convex subset $C$ of a Banach space $E$ and $\lim _{n \rightarrow \infty} t_{n}=\infty$. Using viscosity method, Chen and Song [3] studied the strong convergence of the following iterative method for a nonexpansive semigroup $\{T(t): t \geq 0\}$ with $\operatorname{Fix}(\mathcal{S}) \neq \emptyset$ in a Banach space:

$$
x_{n+1}=\alpha_{n} f(x)+\left(1-\alpha_{n}\right) \frac{1}{t_{n}} \int_{0}^{t_{n}} T(s) x_{n} d s, \quad \forall n \in \mathbb{N},
$$

where $f$ is a contraction. Note however that their iterate $x_{n}$ at step $n$ is constructed through the average of the semigroup over the interval $(0, t)$. Suzuki [4] was the first to introduce again in a Hilbert space the following implicit iteration process:

$$
x_{n}=\alpha_{n} u+\left(1-\alpha_{n}\right) T\left(t_{n}\right) x_{n}, \quad \forall n \in \mathbb{N},
$$

for the nonexpansive semigroup case. In 2002, Benavides et al. [5], in a uniformly smooth Banach space, showed that if $\mathcal{S}$ satisfies an asymptotic regularity condition and $\left\{\alpha_{n}\right\}$ fulfills the control conditions $\lim _{n \rightarrow \infty} \alpha_{n}=0, \sum_{n=1}^{\infty} \alpha_{n}=\infty$, and $\lim _{n \rightarrow \infty} \alpha_{n} / \alpha_{n+1}=0$, then both the implicit iteration process (1.5) and the explicit iteration process (1.6),

$$
x_{n+1}=\alpha_{n} u+\left(1-\alpha_{n}\right) T\left(t_{n}\right) x_{n}, \quad \forall n \in \mathbb{N},
$$

converge to a same point of $F(S)$. In 2005, Xu [6] studied the strong convergence of the implicit iteration process (1.2) and (1.5) in a uniformly convex Banach space which admits a 
weakly sequentially continuous duality mapping. Recently, Chen and He [7] introduced the viscosity approximation process:

$$
x_{n+1}=\alpha_{n} f\left(x_{n}\right)+\left(1-\beta_{n}\right) T\left(t_{n}\right) x_{n}, \quad \forall n \in \mathbb{N},
$$

where $f$ is a contraction and $\left\{\alpha_{n}\right\}$ is a sequence in $(0,1)$ and a nonexpansive semigroup $\{T(t)$ : $t \geq 0\}$. The strong convergence theorem of $\left\{x_{n}\right\}$ is proved in a reflexive Banach space which admits a weakly sequentially continuous duality mapping. In [8], Chen et al. introduced and studied modified Mann iteration for nonexpansive mapping in a uniformly convex Banach space.

On the other hand, iterative approximation methods for nonexpansive mappings have recently been applied to solve convex minimization problems; see, for example, [9-11] and the references therein. Let $H$ be a real Hilbert space, whose inner product and norm are denoted by $\langle\cdot, \cdot\rangle$ and $\|\cdot\|$, respectively. Let $A$ be a strongly positive bounded linear operator on $H$; that is, there is a constant $\bar{\gamma}>0$ with property

$$
\langle A x, x\rangle \geq \bar{\gamma}\|x\|^{2} \quad \forall x \in H .
$$

A typical problem is to minimize a quadratic function over the set of the fixed points of a nonexpansive mapping on a real Hilbert space $H$ :

$$
\min _{x \in C} \frac{1}{2}\langle A x, x\rangle-\langle x, b\rangle
$$

where $C$ is the fixed point set of a nonexpansive mapping $T$ on $H$ and $b$ is a given point in $H$. In 2003, Xu [10] proved that the sequence $\left\{x_{n}\right\}$ defined by the iterative method below, with the initial guess $x_{0} \in H$ chosen arbitrarily,

$$
x_{n+1}=\left(I-\alpha_{n} A\right) T x_{n}+\alpha_{n} u, \quad n \geq 0,
$$

converges strongly to the unique solution of the minimization problem (1.9) provided the sequence $\left\{\alpha_{n}\right\}$ satisfies certain conditions. Using the viscosity approximation method, Moudafi [12] introduced the following iterative process for nonexpansive mappings (see [13] for further developments in both Hilbert and Banach spaces). Let $f$ be a contraction on $H$. Starting with an arbitrary initial $x_{0} \in H$, define a sequence $\left\{x_{n}\right\}$ recursively by

$$
x_{n+1}=\left(1-\alpha_{n}\right) T x_{n}+\alpha_{n} f\left(x_{n}\right), \quad n \geq 0,
$$

where $\left\{\alpha_{n}\right\}$ is a sequence in $(0,1)$. It is proved $[12,13]$ that, under certain appropriate conditions imposed on $\left\{\alpha_{n}\right\}$, the sequence $\left\{x_{n}\right\}$ generated by (1.11) strongly converges to the unique solution $x^{*}$ in $C$ of the variational inequality

$$
\left\langle(I-f) x^{*}, x-x^{*}\right\rangle \geq 0, \quad x \in H .
$$


Recently, Marino and Xu [14] mixed the iterative method (1.10) and the viscosity approximation method (1.11) and considered the following general iterative method:

$$
x_{n+1}=\left(I-\alpha_{n} A\right) T x_{n}+\alpha_{n} \gamma f\left(x_{n}\right), \quad n \geq 0,
$$

where $A$ is a strongly positive bounded linear operator on $H$. They proved that if the sequence $\left\{\alpha_{n}\right\}$ of parameters satisfies the certain conditions, then the sequence $\left\{x_{n}\right\}$ generated by (1.13) converges strongly to the unique solution $x^{*}$ in $H$ of the variational inequality

$$
\left\langle(A-\gamma f) x^{*}, x-x^{*}\right\rangle \geq 0, \quad x \in H
$$

which is the optimality condition for the minimization problem, $\min _{x \in C}(1 / 2)\langle A x, x\rangle-h(x)$, where $h$ is a potential function for $\gamma f$ (i.e., $h^{\prime}(x)=\gamma f(x)$ for $x \in H$ ).

Very recently, Li et al. [15] introduced the following iterative procedures for the approximation of common fixed points of a one-parameter nonexpansive semigroup on a Hilbert space $H$ :

$$
x_{0}=x \in H, \quad x_{n+1}=\left(I-\alpha_{n} A\right) \frac{1}{t_{n}} \int_{0}^{t_{n}} T(s) x_{n} d s+\alpha_{n} \gamma f\left(x_{n}\right), \quad n \geq 0,
$$

where $A$ is a strongly positive bounded linear operator on $H$.

Let $\delta$ and $\lambda$ be two positive real numbers such that $\delta, \lambda<1$. Recall that a mapping $F$ with domain $D(F)$ and range $R(F)$ in $E$ is called $\delta$-strongly accretive if, for each $x, y \in D(F)$, there exists $j(x-y) \in J(x-y)$ such that

$$
\langle F x-F y, j(x-y)\rangle \geq \delta\|x-y\|^{2},
$$

where $J$ is the normalized duality mapping from $E$ into the dual space $E^{*}$. Recall also that a mapping $F$ is called $\lambda$-strictly pseudocontractive if, for each $x, y \in D(F)$, there exists $j(x-y) \in$ $J(x-y)$ such that

$$
\langle F x-F y, j(x-y)\rangle \leq\|x-y\|^{2}-\lambda\|(x-y)-(F x-F y)\|^{2}
$$

It is easy to see that (1.17) can be rewritten as

$$
\langle(I-F) x-(I-F) y, j(x-y)\rangle \geq \lambda\|(I-F) x-(I-F) y\|^{2},
$$

see [16].

In this paper, motivated by the above results, we introduce and study the strong convergence theorems of the general iterative scheme $\left\{x_{n}\right\}$ defined by (1.19) in the framework of a reflexive Banach space $E$ which admits a weakly sequentially continuous duality mapping:

$$
x_{0}=x \in E, \quad x_{n+1}=\alpha_{n} \gamma f\left(x_{n}\right)+\left(I-\alpha_{n} F\right) T\left(t_{n}\right) x_{n}, \quad n \geq 0,
$$


where $F$ is $\delta$-strongly accretive and $\lambda$-strictly pseudocontractive with $\delta+\lambda>1, f$ is a contraction on $E$ with coefficient $0<\alpha<1, \gamma$ is a positive real number such that $\gamma<(1 / \alpha)(1-$ $\sqrt{(1-\delta) / \lambda})$, and $\mathcal{S}=\{T(t): 0 \leq t<\infty\}$ is a nonexpansive semigroup on $E$. The strong convergence theorems are proved under some appropriate control conditions on parameters $\left\{\alpha_{n}\right\}$ and $\left\{t_{n}\right\}$. Furthermore, by using these results, we obtain strong convergence theorems of the following new general iterative schemes $\left\{y_{n}\right\}$ and $\left\{z_{n}\right\}$ defined by

$$
\begin{gathered}
y_{0}=y \in E, \quad y_{n+1}=\alpha_{n} \gamma f\left(T\left(t_{n}\right) y_{n}\right)+\left(I-\alpha_{n} F\right) T\left(t_{n}\right) y_{n}, \quad n \geq 0, \\
z_{0}=z \in E, \quad z_{n+1}=T\left(t_{n}\right)\left(\alpha_{n} \gamma f\left(z_{n}\right)+\left(I-\alpha_{n} F\right) z_{n}\right), \quad n \geq 0 .
\end{gathered}
$$

The results presented in this paper extend and improve the main results in Li et al. [15], Chen and $\mathrm{He}[7]$, and many others.

\section{Preliminaries}

Throughout this paper, it is assumed that $E$ is a real Banach space with norm $\|\cdot\|$ and let $J$ denote the normalized duality mapping from $E$ into $E^{*}$ given by

$$
J(x)=\left\{f \in E^{*}:\langle x, f\rangle=\|x\|^{2}=\|f\|^{2}\right\}
$$

for each $x \in E$, where $E^{*}$ denotes the dual space of $E,\langle\cdot, \cdot\rangle$ denotes the generalized duality pairing, and $\mathbb{N}$ denotes the set of all positive integers. In the sequel, we will denote the single-valued duality mapping by $j$, and consider $F(T)=\{x \in C: T x=x\}$. When $\left\{x_{n}\right\}$ is a sequence in $E$, then $x_{n} \rightarrow x$ (resp., $x_{n} \rightarrow x, x_{n} \stackrel{*}{\rightarrow} x$ ) will denote strong (resp., weak, weak*) convergence of the sequence $\left\{x_{n}\right\}$ to $x$. In a Banach space $E$, the following result (the subdifferential inequality) is well known [17, Theorem 4.2.1]: for all $x, y \in E$, for all $j(x+y) \in J(x+y)$, for all $j(x) \in J(x)$,

$$
\|x\|^{2}+2\langle y, j(x)\rangle \leq\|x+y\|^{2} \leq\|x\|^{2}+\langle y, j(x+y)\rangle
$$

A real Banach space $E$ is said to be strictly convex if $\|x+y\| / 2<1$ for all $x, y \in E$ with $\|x\|=\|y\|=1$ and $x \neq y$. It is said to be uniformly convex if, for all $\epsilon \in[0,2]$, there exits $\delta_{\epsilon}>0$ such that

$$
\|x\|=\|y\|=1 \quad \text { with }\|x-y\| \geq \epsilon \quad \text { implies } \frac{\|x+y\|}{2}<1-\delta_{\epsilon} .
$$

The following results are well known and can be founded in [17]:

(i) a uniformly convex Banach space $E$ is reflexive and strictly convex [17, Theorems 4.2.1 and 4.1.6],

(ii) if $E$ is a strictly convex Banach space and $T: E \rightarrow E$ is a nonexpansive mapping, then fixed point set $F(T)$ of $T$ is a closed convex subset of $E$ [17, Theorem 4.5.3]. 
If a Banach space $E$ admits a sequentially continuous duality mapping $J$ from weak topology to weak star topology, then from Lemma 1 of [18], it follows that the duality mapping $J$ is single-valued and also $E$ is smooth. In this case, duality mapping $J$ is also said to be weakly sequentially continuous, that is, for each $\left\{x_{n}\right\} \subset E$ with $x_{n} \rightarrow x$, then $J\left(x_{n}\right) \stackrel{*}{\rightarrow} J(x)$ (see $[18,19])$.

In the sequel, we will denote the single-valued duality mapping by $j$. A Banach space $E$ is said to satisfy Opial's condition if, for any sequence $\left\{x_{n}\right\}$ in $E, x_{n} \rightarrow x$ as $n \rightarrow \infty$ implies

$$
\limsup _{n \rightarrow \infty}\left\|x_{n}-x\right\|<\limsup _{n \rightarrow \infty}\left\|x_{n}-y\right\| \quad \forall y \in E \text { with } x \neq y \text {. }
$$

By Theorem 1 of [18], we know that if $E$ admits a weakly sequentially continuous duality mapping, then $E$ satisfies Opial's condition and $E$ is smooth; for the details, see [18].

Now, we present the concept of uniformly asymptotically regular semigroup (also see $[20,21])$. Let $C$ be a nonempty closed convex subset of a Banach space $E, \mathcal{S}=\{T(t): 0 \leq$ $t<\infty\}$ a continuous operator semigroup on $C$. Then, $S$ is said to be uniformly asymptotically regular (in short, u.a.r.) on $C$ if, for all $h \geq 0$ and any bounded subset $D$ of $C$,

$$
\lim _{t \rightarrow \infty} \sup _{x \in D}\|T(h)(T(t) x)-T(t) x\|=0 .
$$

The nonexpansive semigroup $\left\{\sigma_{t}: t>0\right\}$ defined by the following lemma is an example of u.a.r. operator semigroup. Other examples of u.a.r. operator semigroup can be found in [20, Examples 17 and 18].

Lemma 2.1 (see [3, Lemma 2.7]). Let $C$ be a nonempty closed convex subset of a uniformly convex Banach space $E, D$ a bounded closed convex subset of $C$, and $S=\{T(s): 0 \leq s<\infty\}$ a nonexpansive semigroup on $C$ such that $F(\mathcal{S}) \neq \emptyset$. For each $h>0$, set $\sigma_{t}(x)=(1 / t) \int_{0}^{t} T(s) x d s$, then

$$
\lim _{t \rightarrow \infty} \sup _{x \in D}\left\|\sigma_{t}(x)-T(h) \sigma_{t}(x)\right\|=0 .
$$

Example 2.2. The set $\left\{\sigma_{t}: t>0\right\}$ defined by Lemma 2.1 is u.a.r. nonexpansive semigroup. In fact, it is obvious that $\left\{\sigma_{t}: t>0\right\}$ is a nonexpansive semigroup. For each $h>0$, we have

$$
\begin{aligned}
\left\|\sigma_{t}(x)-\sigma_{h} \sigma_{t}(x)\right\| & =\left\|\sigma_{t}(x)-\frac{1}{h} \int_{0}^{h} T(s) \sigma_{t}(x) d s\right\| \\
& =\left\|\frac{1}{h} \int_{0}^{h}\left(\sigma_{t}(x)-T(s) \sigma_{t}(x)\right) d s\right\| \\
& \leq \frac{1}{h} \int_{0}^{h}\left\|\sigma_{t}(x)-T(s) \sigma_{t}(x)\right\| d s .
\end{aligned}
$$


Applying Lemma 2.1, we have

$$
\lim _{t \rightarrow \infty} \sup _{x \in D}\left\|\sigma_{t}(x)-\sigma_{h} \sigma_{t}(x)\right\| \leq \frac{1}{h} \int_{0}^{h} \lim _{t \rightarrow \infty} \sup _{x \in D}\left\|\sigma_{t}(x)-T(s) \sigma_{t}(x)\right\| d s=0 .
$$

Let $C$ be a nonempty closed and convex subset of a Banach space $E$ and $D$ a nonempty subset of $C$. A mapping $Q: C \rightarrow D$ is said to be sunny if

$$
Q(Q x+t(x-Q x))=Q x
$$

whenever $Q x+t(x-Q x) \in C$ for $x \in C$ and $t=0$. A mapping $Q: C \rightarrow D$ is called a retraction if $Q x=x$ for all $x \in D$. Furthermore, $Q$ is a sunny nonexpansive retraction from $C$ onto $D$ if $Q$ is a retraction from $C$ onto $D$ which is also sunny and nonexpansive. A subset $D$ of $C$ is called a sunny nonexpansive retraction of $C$ if there exists a sunny nonexpansive retraction from $C$ onto $D$. The following lemma concerns the sunny nonexpansive retraction.

Lemma 2.3 (see $[22,23]$ ). Let $C$ be a closed convex subset of a smooth Banach space E. Let $D$ be a nonempty subset of $C$ and $Q: C \rightarrow D$ be a retraction. Then, $Q$ is sunny and nonexpansive if and only if

$$
\langle u-Q u, j(y-Q u)\rangle \leq 0
$$

for all $u \in C$ and $y \in D$.

Lemma 2.4 (see [24, Lemma 2.3]). Let $\left\{a_{n}\right\}$ be a sequence of nonnegative real numbers satisfying the property

$$
a_{n+1} \leq\left(1-t_{n}\right) a_{n}+t_{n} c_{n}+b_{n}, \quad \forall n \geq 0,
$$

where $\left\{t_{n}\right\},\left\{b_{n}\right\}$, and $\left\{c_{n}\right\}$ satisfy the restrictions

(i) $\sum_{n=1}^{\infty} t_{n}=\infty$;

(ii) $\sum_{n=1}^{\infty} b_{n}<\infty$;

(iii) $\limsup _{n \rightarrow \infty} c_{n} \leq 0$.

Then, $\lim _{n \rightarrow \infty} a_{n}=0$.

The following lemma will be frequently used throughout the paper and can be found in [25].

Lemma 2.5 (see [25, Lemma 2.7]). Let E be a real smooth Banach space and $F: E \rightarrow E$ a mapping.

(i) If $F$ is $\delta$-strongly accretive and $\lambda$-strictly pseudocontractive with $\delta+\lambda>1$, then $I-F$ is contractive with constant $\sqrt{(1-\delta) / \lambda}$.

(i) If $F$ is $\delta$-strongly accretive and $\lambda$-strictly pseudocontractive with $\delta+\lambda>1$, then, for any fixed number $\tau \in(0,1), I-\tau F$ is contractive with constant $1-\tau(1-\sqrt{(1-\delta) / \lambda})$. 


\section{Main Results}

Now, we are in a position to state and prove our main results.

Theorem 3.1. Let $E$ be a reflexive Banach space which admits a weakly sequentially continuous duality mapping $J$. Let $\mathcal{S}=\{T(t): 0 \leq t<\infty\}$ be a u.a.r. nonexpansive semigroup on $E$ such that $\operatorname{Fix}(S) \neq \emptyset$. Suppose that the real sequences $\left\{\alpha_{n}\right\} \subset[0,1],\left\{t_{n}\right\} \subset(0, \infty)$ satisfy the conditions

$$
\lim _{n \rightarrow \infty} \alpha_{n}=0, \quad \sum_{n=0}^{\infty} \alpha_{n}=\infty, \quad \lim _{n \rightarrow \infty} t_{n}=\infty
$$

Let $F$ be $\delta$-strongly accretive and $\lambda$-strictly pseudocontractive with $\delta+\lambda>1, f: E \rightarrow E$ a contraction mapping with coefficient $\alpha \in(0,1)$, and $\gamma$ a positive real number such that $\gamma<(1 / \alpha)(1-$ $\sqrt{(1-\delta) / \lambda})$. Then, the sequence $\left\{x_{n}\right\}$ defined by (1.19) converges strongly to $\tilde{x}$, where $\tilde{x}$ is the unique solution in $\operatorname{Fix}(\mathcal{S})$ of the variational inequality

$$
\langle(F-\gamma f) \tilde{x}, j(x-\tilde{x})\rangle \geq 0, \quad x \in \operatorname{Fix}(S)
$$

or equivalently $\tilde{x}=Q_{\operatorname{Fix}(\mathcal{S})}(I-F+\gamma f) \tilde{x}$, where $Q_{\mathrm{Fix}(\mathcal{S})}$ is the sunny nonexpansive retraction of $E$ onto $\operatorname{Fix}(S)$.

Proof. Note that $\operatorname{Fix}(\mathcal{S})$ is a nonempty closed convex set. We first show that $\left\{x_{n}\right\}$ is bounded. Let $q \in \operatorname{Fix}(\mathcal{S})$. Thus, by Lemma 2.5, we have

$$
\begin{aligned}
\left\|x_{n+1}-q\right\|= & \left\|\alpha_{n} \gamma f\left(x_{n}\right)+\left(I-\alpha_{n} F\right) T\left(t_{n}\right) x_{n}-\left(I-\alpha_{n} F\right) q-\alpha_{n} F q\right\| \\
\leq & \alpha_{n}\left\|\gamma f\left(x_{n}\right)-F q\right\|+\left\|I-\alpha_{n} F\right\|\left\|T\left(t_{n}\right) x_{n}-q\right\| \\
\leq & \alpha_{n} \gamma\left\|f\left(x_{n}\right)-f(q)\right\|+\alpha_{n}\|\gamma f(q)-F q\|+\left\|I-\alpha_{n} F\right\|\left\|x_{n}-q\right\| \\
\leq & \alpha_{n} \alpha \gamma\left\|x_{n}-q\right\|+\alpha_{n}\|\gamma f(q)-F q\| \\
& +\left(1-\alpha_{n}\left(1-\sqrt{\frac{1-\delta}{\lambda}}\right)\right)\left\|x_{n}-q\right\| \\
= & \left(1-\alpha_{n}\left(1-\sqrt{\frac{1-\delta}{\lambda}}-\alpha \gamma\right)\right)\left\|x_{n}-q\right\| \\
& +\alpha_{n}\left(1-\sqrt{\frac{1-\delta}{\lambda}-\alpha \gamma}\right) \frac{\|\gamma f(q)-F q\|}{1-\sqrt{(1-\delta) / \lambda}-\alpha \gamma} \\
\leq & \max \left\{\left\|x_{n}-q\right\|, \frac{1}{1-\sqrt{(1-\delta) / \lambda}-\alpha \gamma}\|\gamma f(q)-F q\|\right\}, \quad \forall n \geq 0 .
\end{aligned}
$$


By induction, we get

$$
\left\|x_{n}-q\right\| \leq \max \left\{\left\|x_{0}-q\right\|, \frac{1}{1-\sqrt{(1-\delta) / \lambda}-\alpha \gamma}\|\gamma f(q)-F q\|\right\}, \quad n \geq 0 .
$$

This implies that $\left\{x_{n}\right\}$ is bounded and, hence, so are $\left\{f\left(x_{n}\right)\right\}$ and $\left\{F T\left(t_{n}\right) x_{n}\right\}$. This implies that

$$
\lim _{n \rightarrow \infty}\left\|x_{n+1}-T\left(t_{n}\right) x_{n}\right\|=\lim _{n \rightarrow \infty} \alpha_{n}\left\|\gamma f\left(x_{n}\right)-F T\left(t_{n}\right) x_{n}\right\|=0
$$

Since $\{T(t)\}$ is a u.a.r. nonexpansive semigroup and $\lim _{n \rightarrow \infty} t_{n}=\infty$, we have, for all $h>0$,

$$
\lim _{n \rightarrow \infty}\left\|T(h)\left(T\left(t_{n}\right) x_{n}\right)-T\left(t_{n}\right) x_{n}\right\| \leq \lim _{n \rightarrow \infty} \sup _{x \in\left\{x_{n}\right\}}\left\|T(h)\left(T\left(t_{n}\right) x\right)-T\left(t_{n}\right) x\right\|=0 .
$$

Hence, for all $h>0$,

$$
\begin{aligned}
\left\|x_{n+1}-T(h) x_{n+1}\right\| & \leq\left\|x_{n+1}-T\left(t_{n}\right) x_{n}\right\|+\left\|T\left(t_{n}\right) x_{n}-T(h) T\left(t_{n}\right) x_{n}\right\|+\left\|T(h) T\left(t_{n}\right) x_{n}-T(h) x_{n+1}\right\| \\
& \leq 2\left\|x_{n+1}-T\left(t_{n}\right) x_{n}\right\|+\left\|T\left(t_{n}\right) x_{n}-T(h) T\left(t_{n}\right) x_{n}\right\| \longrightarrow 0 .
\end{aligned}
$$

That is, for all $h>0$,

$$
\lim _{n \rightarrow \infty}\left\|x_{n}-T(h) x_{n}\right\|=0
$$

Let $\Phi=Q_{\text {Fix }(S)}$. Then, $\Phi(I-F-\gamma f)$ is a contraction on $E$. In fact, from Lemma 2.5(i), we have

$$
\begin{aligned}
\|\Phi(I-F-\gamma f) x-\Phi(I-F-\gamma f) y\| & \leq\|(I-F-\gamma f) x-(I-F-\gamma f) y\| \\
& \leq\|(I-F) x-(I-F) y\|+\gamma\|f(x)-f(y)\| \\
& \leq \sqrt{\frac{1-\delta}{\lambda}}\|x-y\|+\alpha \gamma\|x-y\| \\
& =\left(\sqrt{\frac{1-\delta}{\lambda}}+\alpha \gamma\right)\|x-y\|, \quad \forall x, y \in E .
\end{aligned}
$$

Therefore, $\Phi(I-F-\gamma f)$ is a contraction on $E$ due to $(\sqrt{(1-\delta) / \lambda}+\alpha \gamma) \in(0,1)$. Thus, by Banach contraction principle, $Q_{\operatorname{Fix}(\mathcal{S})}(I-F-\gamma f)$ has a unique fixed point $\tilde{x}$. Then, using Lemma 2.3, $\tilde{x}$ is the unique solution in $\operatorname{Fix}(S)$ of the variational inequality (3.2). Next, we show that

$$
\limsup _{n \rightarrow \infty}\left\langle\gamma f(\tilde{x})-F \tilde{x}, j\left(x_{n}-\tilde{x}\right)\right\rangle \leq 0
$$


Indeed, we can take a subsequence $\left\{x_{n_{k}}\right\}$ of $\left\{x_{n}\right\}$ such that

$$
\limsup _{n \rightarrow \infty}\left\langle\gamma f(\tilde{x})-F \tilde{x}, j\left(x_{n}-\tilde{x}\right)\right\rangle=\lim _{k \rightarrow \infty}\left\langle\gamma f(\tilde{x})-F \tilde{x}, j\left(x_{n_{k}}-\tilde{x}\right)\right\rangle
$$

We may assume that $x_{n_{k}} \rightarrow p \in E$ as $k \rightarrow \infty$, since a Banach space $E$ has a weakly sequentially continuous duality mapping $J$ satisfying Opial's condition [13]. We will prove that $p \in$ $\operatorname{Fix}(\mathcal{S})$. Suppose the contrary, $p \notin \operatorname{Fix}(\mathcal{S})$, that is, $T\left(h_{0}\right) p \neq p$ for some $h_{0}>0$. It follows from (3.8) and Opial's condition that

$$
\begin{aligned}
\liminf _{k \rightarrow \infty}\left\|x_{n_{k}}-p\right\| & <\liminf _{k \rightarrow \infty}\left\|x_{n_{k}}-T\left(h_{0}\right) p\right\| \\
& \leq \liminf _{k \rightarrow \infty}\left\{\left\|x_{n_{k}}-T\left(h_{0}\right) x_{n_{k}}\right\|+\left\|T\left(h_{0}\right) x_{n_{k}}-T\left(h_{0}\right) p\right\|\right\} \\
& \leq \liminf _{k \rightarrow \infty}\left\{\left\|x_{n_{k}}-T\left(h_{0}\right) x_{n_{k}}\right\|+\left\|x_{n_{k}}-p\right\|\right\} \\
& =\liminf _{k \rightarrow \infty}\left\|x_{n_{k}}-p\right\| .
\end{aligned}
$$

This is a contradiction, which shows that $p \in F(T(h))$ for all $h>0$, that is, $p \in \operatorname{Fix}(\mathcal{S})$. In view of the variational inequality (3.2) and the assumption that duality mapping $J$ is weakly sequentially continuous, we conclude

$$
\begin{aligned}
\limsup _{n \rightarrow \infty}\left\langle\gamma f(\tilde{x})-F \tilde{x}, j\left(x_{n}-\tilde{x}\right)\right\rangle & =\lim _{k \rightarrow \infty}\left\langle\gamma f(\tilde{x})-F \tilde{x}, j\left(x_{n_{k}}-\tilde{x}\right)\right\rangle \\
& \leq\langle\gamma f(\tilde{x})-F \tilde{x}, j(p-\tilde{x})\rangle \leq 0 .
\end{aligned}
$$

Finally, we will show that $x_{n} \rightarrow \tilde{x}$. For each $n \geq 0$, we have

$$
\begin{aligned}
\left\|x_{n+1}-\tilde{x}\right\|^{2} & =\left\|\alpha_{n} \gamma f\left(x_{n}\right)+\left(I-\alpha_{n} F\right) T\left(t_{n}\right) x_{n}-\left(I-\alpha_{n} F\right) \tilde{x}-\alpha_{n} F \tilde{x}\right\|^{2} \\
\leq & \left\|\alpha_{n} \gamma f\left(x_{n}\right)-\alpha_{n} F \tilde{x}+\left(I-\alpha_{n} F\right) T\left(t_{n}\right) x_{n}-\left(I-\alpha_{n} F\right) \tilde{x}\right\|^{2} \\
& =\left\|\left(I-\alpha_{n} F\right) T\left(t_{n}\right) x_{n}-\left(I-\alpha_{n} F\right) \tilde{x}\right\|^{2}+2 \alpha_{n}\left\langle\gamma f\left(x_{n}\right)-F \tilde{x}, j\left(x_{n+1}-\tilde{x}\right)\right\rangle \\
\leq & \left(1-\alpha_{n}\left(1-\sqrt{\frac{1-\delta}{\lambda}}\right)\right)^{2}\left\|x_{n}-\tilde{x}\right\|^{2}+2 \alpha_{n}\left\langle\gamma f\left(x_{n}\right)-\gamma f(\tilde{x}), j\left(x_{n+1}-\tilde{x}\right)\right\rangle \\
& +2 \alpha_{n}\left\langle\gamma f(\tilde{x})-F \tilde{x}, j\left(x_{n+1}-\tilde{x}\right)\right\rangle .
\end{aligned}
$$


International Journal of Mathematics and Mathematical Sciences

On the other hand,

$$
\begin{aligned}
& \left\langle\gamma f\left(x_{n}\right)-\gamma f(\tilde{x}), j\left(x_{n+1}-\tilde{x}\right)\right\rangle \\
& \leq \gamma \alpha\left\|x_{n}-\tilde{x}\right\|\left\|x_{n+1}-\tilde{x}\right\| \\
& \leq \gamma \alpha\left\|x_{n}-\tilde{x}\right\|\left[\sqrt{\left.\left(1-\alpha_{n}\left(1-\sqrt{\frac{1-\delta}{\lambda}}\right)\right)^{2}\left\|x_{n}-\tilde{x}\right\|^{2}+2 \alpha_{n}\left|\left\langle\gamma f\left(x_{n}\right)-F \tilde{x}, j\left(x_{n+1}-\tilde{x}\right)\right\rangle\right|\right]}\right] \\
& \leq \gamma \alpha\left(1-\alpha_{n}\left(1-\sqrt{\frac{1-\delta}{\lambda}}\right)\right)\left\|x_{n}-\tilde{x}\right\|^{2} \\
& \quad+\gamma \alpha\left\|x_{n}-\tilde{x}\right\| \sqrt{2\left|\left\langle\gamma f\left(x_{n}\right)-F \tilde{x}, j\left(x_{n+1}-\tilde{x}\right)\right\rangle\right|} \sqrt{\alpha_{n}} \\
& \leq \gamma \alpha\left(1-\alpha_{n}\left(1-\sqrt{\frac{1-\delta}{\lambda}}\right)\right)\left\|x_{n}-\tilde{x}\right\|^{2}+\sqrt{\alpha_{n}} M_{0},
\end{aligned}
$$

where $M_{0}$ is a constant satisfying $M_{0} \geq \gamma \alpha\left\|x_{n}-\tilde{x}\right\| \sqrt{2 \mid\left\langle\gamma f\left(x_{n}\right)-F \tilde{x}, j\left(x_{n+1}-\tilde{x}\right)\right\rangle}$. Substituting (3.15) in (3.14), we obtain

$$
\begin{aligned}
& \left\|x_{n+1}-\tilde{x}\right\|^{2} \leq\left(1-\alpha_{n}\left(1-\sqrt{\frac{1-\delta}{\lambda}}\right)\right)^{2}\left\|x_{n}-\tilde{x}\right\|^{2}+2 \alpha_{n} \gamma \alpha\left(1-\alpha_{n}\left(1-\sqrt{\frac{1-\delta}{\lambda}}\right)\right) \\
& \times\left\|x_{n}-\tilde{x}\right\|^{2}+2 \alpha_{n} \sqrt{\alpha_{n}} M_{0}+2 \alpha_{n}\left\langle\gamma f(\tilde{x})-F \tilde{x}, j\left(x_{n+1}-\tilde{x}\right)\right\rangle \\
& =\left(1-2 \alpha_{n}\left(1-\sqrt{\frac{1-\delta}{\lambda}}\right)+\alpha_{n}^{2}\left(1-\sqrt{\frac{1-\delta}{\lambda}}\right)^{2}\right)\left\|x_{n}-\tilde{x}\right\|^{2} \\
& +2 \alpha_{n} \gamma \alpha\left(1-\alpha_{n}\left(1-\sqrt{\frac{1-\delta}{\lambda}}\right)\right)\left\|x_{n}-\tilde{x}\right\|^{2} \\
& +2 \alpha_{n} \sqrt{\alpha_{n}} M_{0}+2 \alpha_{n}\left\langle\gamma f(\tilde{x})-F \tilde{x}, j\left(x_{n+1}-\tilde{x}\right)\right\rangle \\
& =\left(1-2 \alpha_{n}\left[\left(1-\sqrt{\frac{1-\delta}{\lambda}}\right)-\alpha \gamma+\alpha_{n} \gamma \alpha\left(1-\sqrt{\frac{1-\delta}{\lambda}}\right)\right]\right)\left\|x_{n}-\tilde{x}\right\|^{2} \\
& +\alpha_{n}\left[\alpha_{n}\left(1-\sqrt{\frac{1-\delta}{\lambda}}\right)^{2}\left\|x_{n}-\tilde{x}\right\|^{2}+2 M_{0} \sqrt{\alpha_{n}}+2\left\langle\gamma f(\tilde{x})-F \tilde{x}, j\left(x_{n+1}-\tilde{x}\right)\right\rangle\right] \\
& =\left(1-\alpha_{n} \gamma_{n}\right)\left\|x_{n}-\tilde{x}\right\|^{2}+\alpha_{n} \gamma_{n} \frac{\beta_{n}}{\gamma_{n}},
\end{aligned}
$$


where

$$
\begin{gathered}
\gamma_{n}=2\left[\left(1-\sqrt{\frac{1-\delta}{\lambda}}\right)-\alpha \gamma+\alpha_{n} \gamma \alpha\left(1-\sqrt{\frac{1-\delta}{\lambda}}\right)\right], \\
\beta_{n}=\left[\alpha_{n}\left(1-\sqrt{\frac{1-\delta}{\lambda}}\right)^{2}\left\|x_{n}-\tilde{x}\right\|^{2}+2 M_{0} \sqrt{\alpha_{n}}+2\left\langle\gamma f(\tilde{x})-F \tilde{x}, j\left(x_{n+1}-\tilde{x}\right)\right\rangle\right] .
\end{gathered}
$$

It is easily seen that $\sum_{n=1}^{\infty} \alpha_{n} \gamma_{n}=\infty$. Since $\left\{x_{n}\right\}$ is bounded and $\lim _{n \rightarrow \infty} \alpha_{n}=0$, by (3.46), we obtain lim sup $_{n \rightarrow \infty} \beta_{n} / \gamma_{n} \leq 0$, applying Lemma 2.4 to (3.16) to conclude $x_{n} \rightarrow \tilde{x}$ as $n \rightarrow \infty$. This completes the proof.

Using Theorem 3.1, we obtain the following two strong convergence theorems of new iterative approximation methods for a nonexpansive semigroup $\{T(t): 0 \leq t<\infty\}$.

Corollary 3.2. Let $E$ be a reflexive Banach space which admits a weakly sequentially continuous duality mapping $J$. Let $S=\{T(t): 0 \leq t<\infty\}$ be a u.a.r. nonexpansive semigroup on $E$ such that $\operatorname{Fix}(\mathcal{S}) \neq \emptyset$. Suppose that the real sequences $\left\{\alpha_{n}\right\} \subset[0,1],\left\{t_{n}\right\} \subset(0, \infty)$ satisfy the conditions

$$
\lim _{n \rightarrow \infty} \alpha_{n}=0, \quad \sum_{n=0}^{\infty} \alpha_{n}=\infty, \quad \lim _{n \rightarrow \infty} t_{n}=\infty
$$

Let $F$ be $\delta$-strongly accretive and $\lambda$-strictly pseudocontractive with $\delta+\lambda>1, f: E \rightarrow E$ a contraction mapping with coefficient $\alpha \in(0,1)$, and $\gamma$ a positive real number such that $\gamma<(1 / \alpha)(1-$ $\sqrt{(1-\delta) / \lambda})$. Then, the sequence $\left\{y_{n}\right\}$ defined by (1.20) converges strongly to $\tilde{x}$, where $\tilde{x}$ is the unique solution in $\operatorname{Fix}(\mathcal{S})$ of the variational inequality

$$
\langle(F-\gamma f) \tilde{x}, j(x-\tilde{x})\rangle \geq 0, \quad x \in \operatorname{Fix}(\mathcal{S})
$$

or equivalently $\tilde{x}=Q_{\operatorname{Fix}(\mathcal{S})}(I-F+\gamma f) \tilde{x}$, where $Q_{\mathrm{Fix}(\mathcal{S})}$ is the sunny nonexpansive retraction of $E$ onto $\operatorname{Fix}(\mathcal{S})$.

Proof. Let $\left\{x_{n}\right\}$ be the sequence given by $x_{0}=y_{0}$ and

$$
x_{n+1}=\alpha_{n} \gamma f\left(x_{n}\right)+\left(I-\alpha_{n} F\right) T\left(t_{n}\right) x_{n}, \quad \forall n \geq 0 .
$$

Form Theorem 3.1, $x_{n} \rightarrow \tilde{x}$. We claim that $y_{n} \rightarrow \tilde{x}$. Indeed, we estimate

$$
\begin{aligned}
& \left\|x_{n+1}-y_{n+1}\right\| \\
& \quad \leq \alpha_{n} \gamma\left\|f\left(T\left(t_{n}\right) y_{n}\right)-f\left(x_{n}\right)\right\|+\left\|I-\alpha_{n} F\right\|\left\|T\left(t_{n}\right) x_{n}-T\left(t_{n}\right) y_{n}\right\| \\
& \quad \leq \alpha_{n} \gamma \alpha\left\|T\left(t_{n}\right) y_{n}-x_{n}\right\|+\left(1-\alpha_{n}\left(1-\sqrt{\frac{1-\delta}{\ell}}\right)\right)\left\|x_{n}-y_{n}\right\|
\end{aligned}
$$


International Journal of Mathematics and Mathematical Sciences

$$
\begin{aligned}
\leq & \alpha_{n} \gamma \alpha\left\|T\left(t_{n}\right) y_{n}-T\left(t_{n}\right) \tilde{x}\right\|+\alpha_{n} \gamma \alpha\left\|T\left(t_{n}\right) \tilde{x}-x_{n}\right\|+\left(1-\alpha_{n}\left(1-\sqrt{\frac{1-\delta}{\lambda}}\right)\right)\left\|x_{n}-y_{n}\right\| \\
\leq & \alpha_{n} \gamma \alpha\left\|y_{n}-\tilde{x}\right\|+\alpha_{n} \gamma \alpha\left\|\tilde{x}-x_{n}\right\|+\left(1-\alpha_{n}\left(1-\sqrt{\frac{1-\delta}{\lambda}}\right)\right)\left\|x_{n}-y_{n}\right\| \\
\leq & \alpha_{n} \gamma \alpha\left\|y_{n}-x_{n}\right\|+\alpha_{n} \gamma \alpha\left\|x_{n}-\tilde{x}\right\|+\alpha_{n} \gamma \alpha\left\|\tilde{x}-x_{n}\right\|+\left(1-\alpha_{n}\left(1-\sqrt{\frac{1-\delta}{\lambda}}\right)\right)\left\|x_{n}-y_{n}\right\| \\
= & \left(1-\alpha_{n}\left(1-\sqrt{\frac{1-\delta}{\lambda}}-\gamma \alpha\right)\right)\left\|x_{n}-y_{n}\right\| \\
& +\alpha_{n}\left(1-\sqrt{\frac{1-\delta}{\lambda}}-\gamma \alpha\right) \frac{2 \alpha \gamma}{(1-\sqrt{(1-\delta) / \lambda}-\gamma \alpha)}\left\|\tilde{x}-x_{n}\right\| .
\end{aligned}
$$

It follows from $\sum_{n=1}^{\infty} \alpha_{n}=\infty, \lim _{n \rightarrow \infty}\left\|x_{n}-\tilde{x}\right\|=0$, and Lemma 2.4 that $\left\|x_{n}-y_{n}\right\| \rightarrow 0$. Consequently, $y_{n} \rightarrow \tilde{x}$ as required.

Corollary 3.3. Let $E$ be a reflexive Banach space which admits a weakly sequentially continuous duality mapping $J$. Let $\mathcal{S}=\{T(t): 0 \leq t<\infty\}$ be a u.a.r. nonexpansive semigroup on $E$ such that $\operatorname{Fix}(\mathcal{S}) \neq \emptyset$. Suppose that the real sequences $\left\{\alpha_{n}\right\} \subset[0,1],\left\{t_{n}\right\} \subset(0, \infty)$ satisfy the conditions

$$
\lim _{n \rightarrow \infty} \alpha_{n}=0, \quad \sum_{n=0}^{\infty} \alpha_{n}=\infty, \quad \lim _{n \rightarrow \infty} t_{n}=\infty
$$

Let $F$ be $\delta$-strongly accretive and $\lambda$-strictly pseudocontractive with $\delta+\lambda>1, f: E \rightarrow E$ acontraction mapping with coefficient $\alpha \in(0,1)$, and $\gamma$ a positive real number such that $\gamma<(1 / \alpha)(1-$ $\sqrt{(1-\delta) / \lambda})$. Then, the sequence $\left\{z_{n}\right\}$ defined by $(1.21)$ converges strongly to $\tilde{x}$, where $\tilde{x}$ is the unique solution in $\operatorname{Fix}(S)$ of the variational inequality

$$
\langle(F-\gamma f) \tilde{x}, j(x-\tilde{x})\rangle \geq 0, \quad x \in \operatorname{Fix}(\mathcal{S})
$$

or equivalently $\tilde{x}=Q_{\operatorname{Fix}(\mathcal{S})}(I-F+\gamma f) \tilde{x}$, where $Q_{\operatorname{Fix}(\mathcal{S})}$ is the sunny nonexpansive retraction of $E$ onto $\operatorname{Fix}(\mathcal{S})$.

Proof. Define the sequences $\left\{y_{n}\right\}$ and $\left\{\beta_{n}\right\}$ by

$$
y_{n}=\alpha_{n} \gamma f\left(z_{n}\right)+\left(I-\alpha_{n} F\right) z_{n}, \quad \beta_{n}=\alpha_{n+1} \quad \forall n \in \mathbb{N} .
$$


Taking $p \in \operatorname{Fix}(\mathcal{S})$, we have

$$
\begin{aligned}
\left\|z_{n+1}-p\right\| & =\left\|T\left(t_{n}\right) y_{n}-T\left(t_{n}\right) p\right\| \leq\left\|y_{n}-p\right\| \\
& =\left\|\alpha_{n} \gamma f\left(z_{n}\right)+\left(I-\alpha_{n} F\right) z_{n}-\left(I-\alpha_{n} F\right) p-\alpha_{n} F p\right\| \\
& \leq\left(1-\alpha_{n}\left(1-\sqrt{\frac{1-\delta}{\lambda}}\right)\right)\left\|z_{n}-p\right\|+\alpha_{n}\left\|\gamma f\left(z_{n}\right)-F(p)\right\| \\
& =\left(1-\alpha_{n}\left(1-\sqrt{\frac{1-\delta}{\lambda}}\right)\right)\left\|z_{n}-p\right\|+\alpha_{n}\left(1-\sqrt{\frac{1-\delta}{\lambda}}\right) \frac{\left\|\gamma f\left(z_{n}\right)-F(p)\right\|}{(1-\sqrt{(1-\delta) / \lambda})} .
\end{aligned}
$$

It follows from induction that

$$
\left\|z_{n+1}-p\right\| \leq \max \left\{\left\|z_{0}-p\right\|, \frac{\left\|\gamma f\left(z_{0}\right)-F(p)\right\|}{1-\sqrt{(1-\delta) / \lambda}}\right\}, \quad n \geq 0 .
$$

Thus, both $\left\{z_{n}\right\}$ and $\left\{y_{n}\right\}$ are bounded. We observe that

$$
y_{n+1}=\alpha_{n+1} \gamma f\left(z_{n+1}\right)+\left(I-\alpha_{n+1} F\right) z_{n+1}=\beta_{n} \gamma f\left(T\left(t_{n}\right) y_{n}\right)+\left(I-\beta_{n} F\right) T\left(t_{n}\right) y_{n}
$$

Thus, Corollary 3.2 implies that $\left\{y_{n}\right\}$ converges strongly to some point $\tilde{x}$. In this case, we also have

$$
\left\|z_{n}-\tilde{x}\right\| \leq\left\|z_{n}-y_{n}\right\|+\left\|y_{n}-\tilde{x}\right\|=\alpha_{n}\left\|\gamma f\left(z_{n}\right)-F z_{n}\right\|+\left\|y_{n}-\tilde{x}\right\| \longrightarrow 0 .
$$

Hence, the sequence $\left\{z_{n}\right\}$ converges strongly to some point $\tilde{x}$. This complete the proof.

Using Theorem 3.1, Lemma 2.1, and Example 2.2, we have the following result.

Corollary 3.4. Let E be a uniformly convex Banach space which admits a weakly sequentially continuous duality mapping $J$. Let $\mathcal{S}=\{T(t): 0 \leq t<\infty\}$ be a nonexpansive semigroup on $E$ such that $\operatorname{Fix}(S) \neq \emptyset$. Suppose that the real sequences $\left\{\alpha_{n}\right\} \subset[0,1],\left\{t_{n}\right\} \subset(0, \infty)$ satisfy the conditions

$$
\lim _{n \rightarrow \infty} \alpha_{n}=0, \quad \sum_{n=0}^{\infty} \alpha_{n}=\infty, \quad \lim _{n \rightarrow \infty} t_{n}=\infty .
$$

Let $F$ be $\delta$-strongly accretive and $\lambda$-strictly pseudocontractive with $\delta+\lambda>1, f: E \rightarrow E$ a contraction mapping with coefficient $\alpha \in(0,1)$, and $\gamma$ a positive real number such that $\gamma<(1 / \alpha)(1-$ $\sqrt{(1-\delta) / \lambda})$. Then, the sequence $\left\{x_{n}\right\}$ defined by

$$
\begin{gathered}
x_{0}=x \in E, \\
x_{n+1}=\alpha_{n} \gamma f\left(x_{n}\right)+\left(I-\alpha_{n} F\right) \frac{1}{t_{n}} \int_{0}^{t_{n}} T(t) x_{n} d s, \quad n \geq 0
\end{gathered}
$$


converges strongly to $\tilde{x}$, where $\tilde{x}$ is the unique solution in $\operatorname{Fix}(\mathcal{S})$ of the variational inequality

$$
\langle(F-r f) \tilde{x}, j(x-\tilde{x})\rangle \geq 0, \quad x \in \operatorname{Fix}(\mathcal{S})
$$

or equivalently $\tilde{x}=Q_{\operatorname{Fix}(\mathcal{S})}((I-F+\gamma f) \tilde{x})$, where $Q_{\operatorname{Fix}(\mathcal{S})}$ is the sunny nonexpansive retraction of $E$ onto $\operatorname{Fix}(\mathcal{S})$.

Corollary 3.5. Let $H$ be a real Hilbert space. Let $\mathcal{S}=\{T(t): 0 \leq t<\infty\}$ be a nonexpansive semigroup on $H$ such that $\operatorname{Fix}(\mathcal{S}) \neq \emptyset$. Suppose that the real sequences $\left\{\alpha_{n}\right\} \subset[0,1],\left\{t_{n}\right\} \subset(0, \infty)$ satisfy the conditions

$$
\lim _{n \rightarrow \infty} \alpha_{n}=0, \quad \sum_{n=0}^{\infty} \alpha_{n}=\infty, \quad \lim _{n \rightarrow \infty} t_{n}=\infty
$$

Let $f: E \rightarrow$ E be a contraction mapping with coefficient $\alpha \in(0,1)$ and A a strongly positive bounded linear operator with coefficient $\bar{\gamma}>1 / 2$ and $0<\gamma<(1-\sqrt{2-2 \bar{\gamma}}) / \alpha$. Then, the sequence $\left\{x_{n}\right\}$ defined by

$$
\begin{gathered}
x_{0}=x \in E, \\
x_{n+1}=\alpha_{n} \gamma f\left(x_{n}\right)+\left(I-\alpha_{n} A\right) \frac{1}{t_{n}} \int_{0}^{t_{n}} T(t) x_{n} d s, \quad n \geq 0
\end{gathered}
$$

converges strongly to $\tilde{x}$, where $\tilde{x}$ is the unique solution in $\operatorname{Fix}(\mathcal{S})$ of the variational inequality

$$
\langle(A-\gamma f) \tilde{x}, j(x-\tilde{x})\rangle \geq 0, \quad x \in \operatorname{Fix}(\mathcal{S})
$$

or equivalently $\tilde{x}=Q_{\operatorname{Fix}(\mathcal{S})}((I-A+\gamma f) \tilde{x})$, where $Q_{\operatorname{Fix}(\mathcal{S})}$ is the sunny nonexpansive retraction of $E$ onto $\operatorname{Fix}(\mathcal{S})$.

Proof. Since $A$ is a strongly positive bounded linear operator with coefficient $\bar{\gamma}$, we have

$$
\langle A x-A y, x-y\rangle \geq \bar{\gamma}\|x-y\|^{2}
$$

Therefore, $A$ is $\bar{\gamma}$-strongly accretive. On the other hand,

$$
\begin{aligned}
\|(I-A) x-(I-A) y\|^{2} & =\langle(x-y)-(A x-A y),(x-y)-(A x-A y)\rangle \\
& =\langle x-y, x-y\rangle-2\langle A x-A y, x-y\rangle+\langle A x-A y, A x-A y\rangle \\
& =\|x-y\|^{2}-2\langle A x-A y, x-y\rangle+\|A x-A y\|^{2} \\
& \leq\|x-y\|^{2}-2\langle A x-A y, x-y\rangle+\|A\|^{2}\|x-y\|^{2} .
\end{aligned}
$$


Since $A$ is strongly positive if and only if $(1 /\|A\|) A$ is strongly positive, we may assume, without loss of generality, that $\|A\|=1$, so that

$$
\begin{aligned}
\langle A x-A y, x-y\rangle & \leq\|x-y\|^{2}-\frac{1}{2}\|(I-A) x-(I-A) y\|^{2} \\
& =\|x-y\|^{2}-\frac{1}{2}\|(x-y)-(A x-A y)\|^{2} .
\end{aligned}
$$

Hence, $A$ is 12-strongly pseudocontractive. Applying Corollary 3.4, we conclude the result.

Theorem 3.6. Let $E$ be a reflexive Banach space which admits a weakly sequentially continuous duality mapping $J$. Let $\mathcal{S}=\{T(t): 0<t<\infty\}$ be a u.a.r. nonexpansive semigroup on $E$ such that $\operatorname{Fix}(\mathcal{S}) \neq \emptyset$. Let $\left\{\alpha_{n}\right\}$ and $\left\{t_{n}\right\}$ be sequences of real number satisfying

$$
0<\alpha_{n}<1, \quad \sum_{n=0}^{\infty} \alpha_{n}=\infty, \quad t_{n}>0, \quad \lim _{n \rightarrow \infty} \alpha_{n}=\lim _{n \rightarrow \infty} \frac{\alpha_{n}}{t_{n}}=0
$$

Let $F$ be $\delta$-strongly accretive and $\lambda$-strictly pseudocontractive with $\delta+\lambda>1, f: E \rightarrow E$ a contraction mapping with coefficient $\alpha \in(0,1)$, and $\gamma$ a positive real number such that $\gamma<(1 / \alpha)(1-$ $\sqrt{(1-\delta) / \lambda})$. Then, the sequence $\left\{x_{n}\right\}$ defined by

$$
\begin{gathered}
x_{0}=x \in E, \\
x_{n+1}=\alpha_{n} \gamma f\left(x_{n}\right)+\left(I-\alpha_{n} F\right) T\left(t_{n}\right) x_{n}, \quad n \geq 0
\end{gathered}
$$

converges strongly to $\tilde{x}$, where $\tilde{x}$ is the unique solution in $\operatorname{Fix}(\mathcal{S})$ of the variational inequality

$$
\langle(F-\gamma f) \tilde{x}, j(x-\tilde{x})\rangle \geq 0, \quad x \in \operatorname{Fix}(\mathcal{S})
$$

or equivalently $\tilde{x}=Q_{\mathrm{Fix}(S)}(I-F+\gamma f) \tilde{x}$, where $Q_{\mathrm{Fix}(S)}$ is the sunny nonexpansive retraction of $E$ onto $\operatorname{Fix}(S)$.

Proof. By the same argument as in the proof of Theorem 3.1, we can obtain that $\left\{x_{n}\right\},\left\{f\left(x_{n}\right)\right\}$, and $\left\{F T\left(t_{n}\right) x_{n}\right\}$ are bounded and $Q_{\operatorname{Fix}(\mathcal{S})}(I-F-\gamma f)$ is a contraction on $E$. Thus, by Banach contraction principle, $Q_{\operatorname{Fix}(\mathcal{S})}(I-F-\gamma f)$ has a unique fixed point $\tilde{x}$. Then, using Lemma 2.3, $\tilde{x}$ is the unique solution in $\operatorname{Fix}(\mathcal{S})$ of the variational inequality (3.40). Next, we show that

$$
\limsup _{n \rightarrow \infty}\left\langle\gamma f(\tilde{x})-F \tilde{x}, j\left(x_{n}-\tilde{x}\right)\right\rangle \leq 0
$$

Indeed, we can take a subsequence $\left\{x_{n_{k}}\right\}$ of $\left\{x_{n}\right\}$ such that

$$
\limsup _{n \rightarrow \infty}\left\langle\gamma f(\tilde{x})-F \tilde{x}, j\left(x_{n}-\tilde{x}\right)\right\rangle=\lim _{k \rightarrow \infty}\left\langle\gamma f(\tilde{x})-F \tilde{x}, j\left(x_{n_{k}}-\tilde{x}\right)\right\rangle .
$$


We may assume that $x_{n_{k}} \rightarrow p \in E$ as $k \rightarrow \infty$. Now, we show that $p \in \operatorname{Fix}(\mathcal{S})$. Put

$$
x_{k}=x_{n_{k}}, \quad \alpha_{k}=\alpha_{n_{k}} \quad s_{k}=t_{n_{k}} \quad \forall k \in \mathbb{N} .
$$

Fix $t>0$, then we have

$$
\begin{aligned}
\left\|x_{k}-T(t) p\right\|= & \sum_{i=0}^{\left[t / s_{i}\right]-1}\left\|T\left((i+1) s_{k}\right) x_{k}-T\left(i s_{k}\right) x_{k}\right\| \\
& +\left\|T\left(\left[\frac{t}{s_{k}}\right] s_{k}\right) x_{k}-T\left(\left[\frac{t}{s_{k}}\right] s_{k}\right) p\right\|+\left\|T\left(\left[\frac{t}{s_{k}}\right] s_{k}\right) p-T(t) p\right\| \\
\leq & {\left[\frac{t}{s_{k}}\right]\left\|T\left(s_{k}\right) x_{k}-x_{k+1}\right\|+\left\|x_{k+1}-p\right\|+\left\|T\left(t-\left[\frac{t}{s_{k}}\right] s_{k}\right) p-p\right\| } \\
\leq & {\left[\frac{t}{s_{k}}\right] \alpha_{k}\left\|F T\left(s_{k}\right) x_{k}-f\left(x_{k}\right)\right\|+\left\|x_{k+1}-p\right\|+\left\|T\left(t-\left[\frac{t}{s_{k}}\right] s_{k}\right) p-p\right\| } \\
\leq & \left(\frac{t \alpha_{k}}{s_{k}}\right)\left\|F T\left(s_{k}\right) x_{k}-f\left(x_{k}\right)\right\|+\left\|x_{k+1}-p\right\|+\max \left\{\|T(s) p-p\|: 0 \leq s \leq s_{k}\right\} .
\end{aligned}
$$

Thus, for all $k \in \mathbb{N}$, we obtain

$$
\limsup _{k \rightarrow \infty}\left\|x_{k}-T(t) p\right\| \leq \limsup _{k \rightarrow \infty}\left\|x_{k+1}-p\right\|=\limsup _{k \rightarrow \infty}\left\|x_{k}-p\right\| .
$$

Since Banach space $E$ has a weakly sequentially continuous duality mapping satisfying Opial's condition [13], we can conclude that $T(t) p=p$ for all $t>0$, that is, $p \in \operatorname{Fix}(\mathcal{S})$. In view of the variational inequality (3.2) and the assumption that duality mapping $J$ is weakly sequentially continuous, we conclude

$$
\begin{aligned}
\limsup _{n \rightarrow \infty}\left\langle\gamma f(\tilde{x})-F \tilde{x}, j\left(x_{n}-\tilde{x}\right)\right\rangle & =\lim _{k \rightarrow \infty}\left\langle\gamma f(\tilde{x})-F \tilde{x}, j\left(x_{n_{k}}-\tilde{x}\right)\right\rangle \\
& \leq\langle\gamma f(\tilde{x})-F \tilde{x}, J(p-\tilde{x})\rangle \leq 0 .
\end{aligned}
$$

By the same argument as in the proof of Theorem 3.1, we conclude that $x_{n} \rightarrow \tilde{x}$ as $n \rightarrow \infty$. This completes the proof.

Using Theorem 3.6 and the method as in the proof of Corollary 3.7, we have the following result.

Corollary 3.7. Let E be a reflexive Banach space which admits a weakly sequentially continuous duality mapping $J$. Let $\mathcal{S}=\{T(t): 0<t<\infty\}$ be a u.a.r. nonexpansive semigroup on $E$ such that $\operatorname{Fix}(\mathcal{S}) \neq \emptyset$. Let $\left\{\alpha_{n}\right\}$ and $\left\{t_{n}\right\}$ be sequences of real number satisfying

$$
0<\alpha_{n}<1, \quad \sum_{n=0}^{\infty} \alpha_{n}=\infty, \quad t_{n}>0, \quad \lim _{n \rightarrow \infty} \alpha_{n}=\lim _{n \rightarrow \infty} \frac{\alpha_{n}}{t_{n}}=0 .
$$


Let $F$ be a $\delta$-strongly accretive and $\lambda$-strictly pseudocontractive with $\delta+\lambda>1, f: E \rightarrow E$ a contraction mapping with coefficient $\alpha \in(0,1)$, and $\gamma$ is a positive real number such that $\gamma<1 / \alpha(1-$ $\sqrt{(1-\delta) / \lambda})$. Then, the sequence $\left\{y_{n}\right\}$ defined by

$$
\begin{gathered}
y_{0}=y \in E, \\
y_{n+1}=\alpha_{n} \gamma f\left(T\left(t_{n}\right) y_{n}\right)+\left(I-\alpha_{n} F\right) T\left(t_{n}\right) y_{n}, \quad n \geq 0
\end{gathered}
$$

converges strongly to $\tilde{x}$, where $\tilde{x}$ is the unique solution in $\operatorname{Fix}(\mathcal{S})$ of the variational inequality

$$
\langle(F-\gamma f) \tilde{x}, j(x-\tilde{x})\rangle \geq 0, \quad x \in \operatorname{Fix}(\mathcal{S})
$$

or equivalently $\tilde{x}=Q_{\mathrm{Fix}(\mathcal{S})}(I-F+\gamma f) \tilde{x}$, where $Q_{\mathrm{Fix}(\mathcal{S})}$ is the sunny nonexpansive retraction of $E$ onto $\operatorname{Fix}(\mathcal{S})$.

Using Theorem 3.6 and the method as in the proof of Corollary 3.8, we have the following result.

Corollary 3.8. Let $E$ be a reflexive Banach space which admits a weakly sequentially continuous duality mapping $J$. Let $\mathcal{S}=\{T(t): 0<t<\infty\}$ be a u.a.r. nonexpansive semigroup on $E$ such that $\operatorname{Fix}(\mathcal{S}) \neq \emptyset$. Let $\left\{\alpha_{n}\right\}$ and $\left\{t_{n}\right\}$ be sequences of real number satisfying

$$
0<\alpha_{n}<1, \quad \sum_{n=0}^{\infty} \alpha_{n}=\infty, \quad t_{n}>0, \quad \lim _{n \rightarrow \infty} \alpha_{n}=\lim _{n \rightarrow \infty} \frac{\alpha_{n}}{t_{n}}=0
$$

Let $F$ be a $\delta$-strongly accretive and $\lambda$-strictly pseudocontractive with $\delta+\lambda>1, f: E \rightarrow E$ a contraction mapping with coefficient $\alpha \in(0,1)$, and $\gamma$ is a positive real number such that $\gamma<(1 / \alpha)(1-$ $\sqrt{(1-\delta) / \lambda})$. Then, the sequence $\left\{z_{n}\right\}$ defined by

$$
\begin{gathered}
z_{0}=z \in E, \\
z_{n+1}=T\left(t_{n}\right)\left(\alpha_{n} \gamma f\left(z_{n}\right)+\left(I-\alpha_{n} F\right) z_{n}\right), \quad n \geq 0
\end{gathered}
$$

converges strongly to $\tilde{x}$, where $\tilde{x}$ is the unique solution in $\operatorname{Fix}(\mathcal{S})$ of the variational inequality

$$
\langle(F-\gamma f) \tilde{x}, j(x-\tilde{x})\rangle \geq 0, \quad x \in \operatorname{Fix}(\mathcal{S})
$$

or equivalently $\tilde{x}=Q_{\operatorname{Fix}(\mathcal{S})}(I-F+\gamma f) \tilde{x}$, where $Q_{\mathrm{Fix}(\mathcal{S})}$ is the sunny nonexpansive retraction of $E$ onto $\operatorname{Fix}(S)$.

Using Theorem 3.6, Lemma 2.1, and Example 2.2, we have the following result. 
Corollary 3.9. Let $E$ be a uniformly convex Banach space which admits a weakly sequentially continuous duality mapping $J$. Let $\mathcal{S}=\{T(t): 0<t<\infty\}$ be a nonexpansive semigroup on $E$ such that $\operatorname{Fix}(S) \neq \emptyset$. Let $\left\{\alpha_{n}\right\}$ and $\left\{t_{n}\right\}$ be sequences of real numbers satisfying

$$
0<\alpha_{n}<1, \quad \sum_{n=0}^{\infty} \alpha_{n}=\infty, \quad t_{n}>0, \quad \lim _{n \rightarrow \infty} \alpha_{n}=\lim _{n \rightarrow \infty} \frac{\alpha_{n}}{t_{n}}=0 .
$$

Let $F$ be $\delta$-strongly accretive and $\lambda$-strictly pseudocontractive with $\delta+\lambda>1, f: E \rightarrow E$ a contraction mapping with coefficient $\alpha \in(0,1)$, and $\gamma$ a positive real number such that $\gamma<(1 / \alpha)(1-$ $\sqrt{(1-\delta) / \lambda})$. Then, the sequence $\left\{x_{n}\right\}$ defined by

$$
\begin{gathered}
x_{0}=x \in E, \\
x_{n+1}=\alpha_{n} \gamma f\left(x_{n}\right)+\left(I-\alpha_{n} F\right) \frac{1}{t_{n}} \int_{0}^{t_{n}} T(t) x_{n} d s, \quad n \geq 0
\end{gathered}
$$

converges strongly to $\tilde{x}$, where $\tilde{x}$ is the unique solution in $\operatorname{Fix}(\mathcal{S})$ of the variational inequality

$$
\langle(F-\gamma f) \tilde{x}, j(x-\tilde{x})\rangle \geq 0, \quad x \in \operatorname{Fix}(\mathcal{S})
$$

or equivalently $\tilde{x}=Q_{\operatorname{Fix}(\mathcal{S})}(I-F+\gamma f) \tilde{x}$, where $Q_{\mathrm{Fix}(\mathcal{S})}$ is the sunny nonexpansive retraction of $E$ onto $\operatorname{Fix}(\mathcal{S})$.

Corollary 3.10. Let $H$ be a real Hilbert space. Let $\mathcal{S}=\{T(t): 0 \leq t<\infty\}$ be a nonexpansive semigroup on $H$ such that $\operatorname{Fix}(\mathcal{S}) \neq \emptyset$. Suppose that the real sequences $\left\{\alpha_{n}\right\} \subset[0,1],\left\{t_{n}\right\} \subset(0, \infty)$ satisfy the conditions

$$
0<\alpha_{n}<1, \quad \sum_{n=0}^{\infty} \alpha_{n}=\infty, \quad t_{n}>0, \quad \lim _{n \rightarrow \infty} \alpha_{n}=\lim _{n \rightarrow \infty} \frac{\alpha_{n}}{t_{n}}=0 .
$$

Let $f: E \rightarrow E$ be a contraction mapping with coefficient $\alpha \in(0,1)$ and $A$ a strongly positive bounded linear operator with coefficient $\bar{\gamma}>1 / 2$ and $0<\gamma<(1-\sqrt{2-2 \bar{\gamma}}) / \alpha$. Then, the sequence $\left\{x_{n}\right\}$ defined by

$$
\begin{gathered}
x_{0}=x \in E, \\
x_{n+1}=\alpha_{n} \gamma f\left(x_{n}\right)+\left(I-\alpha_{n} A\right) \frac{1}{t_{n}} \int_{0}^{t_{n}} T(t) x_{n} d s, \quad n \geq 0
\end{gathered}
$$

converges strongly to $\tilde{x}$, where $\tilde{x}$ is the unique solution in $\operatorname{Fix}(\mathcal{S})$ of the variational inequality

$$
\langle(A-\gamma f) \tilde{x}, j(x-\tilde{x})\rangle \geq 0, \quad x \in \operatorname{Fix}(\mathcal{S})
$$

or equivalently $\tilde{x}=Q_{\mathrm{Fix}(\mathcal{S})}((I-A+\gamma f) \tilde{x})$, where $Q_{\mathrm{Fix}(S)}$ is the sunny nonexpansive retraction of $E$ onto $\operatorname{Fix}(\mathcal{S})$. 


\section{Acknowledgment}

The project was supported by the "Centre of Excellence in Mathematics" under the Commission on Higher Education, Ministry of Education, Thailand.

\section{References}

[1] N. Shioji and W. Takahashi, "Strong convergence theorems for asymptotically nonexpansive semigroups in Hilbert spaces," Nonlinear Analysis: Theory, Methods \& Applications, vol. 34, no. 1, pp. 87-99, 1998.

[2] T. Shimizu and W. Takahashi, "Strong convergence to common fixed points of families of nonexpansive mappings," Journal of Mathematical Analysis and Applications, vol. 211, no. 1, pp. 71-83, 1997.

[3] R. Chen and Y. Song, "Convergence to common fixed point of nonexpansive semigroups," Journal of Computational and Applied Mathematics, vol. 200, no. 2, pp. 566-575, 2007.

[4] T. Suzuki, "On strong convergence to common fixed points of nonexpansive semigroups in Hilbert spaces," Proceedings of the American Mathematical Society, vol. 131, no. 7, pp. 2133-2136, 2003.

[5] T. D. Benavides, G. López Acedo, and H.-K. Xu, "Construction of sunny nonexpansive retractions in Banach spaces," Bulletin of the Australian Mathematical Society, vol. 66, no. 1, pp. 9-16, 2002.

[6] H.-K. Xu, "A strong convergence theorem for contraction semigroups in Banach spaces," Bulletin of the Australian Mathematical Society, vol. 72, no. 3, pp. 371-379, 2005.

[7] R. Chen and H. He, "Viscosity approximation of common fixed points of nonexpansive semigroups in Banach space," Applied Mathematics Letters, vol. 20, no. 7, pp. 751-757, 2007.

[8] R. D. Chen, H. M. He, and M. A. Noor, "Modified Mann iterations for nonexpansive semigroups in Banach space," Acta Mathematica Sinica, vol. 26, no. 1, pp. 193-202, 2010.

[9] F. Deutsch and I. Yamada, "Minimizing certain convex functions over the intersection of the fixed point sets of nonexpansive mappings," Numerical Functional Analysis and Optimization. An International Journal, vol. 19, no. 1-2, pp. 33-56, 1998.

[10] H. K. Xu, "An iterative approach to quadratic optimization," Journal of Optimization Theory and Applications, vol. 116, no. 3, pp. 659-678, 2003.

[11] H.-K. Xu, "Iterative algorithms for nonlinear operators," Journal of the London Mathematical Society. Second Series, vol. 66, no. 1, pp. 240-256, 2002.

[12] A. Moudafi, "Viscosity approximation methods for fixed-points problems," Journal of Mathematical Analysis and Applications, vol. 241, no. 1, pp. 46-55, 2000.

[13] H.-K. Xu, "Approximations to fixed points of contraction semigroups in Hilbert spaces," Numerical Functional Analysis and Optimization, vol. 19, no. 1-2, pp. 157-163, 1998.

[14] G. Marino and H.-K. Xu, "A general iterative method for nonexpansive mappings in Hilbert spaces," Journal of Mathematical Analysis and Applications, vol. 318, no. 1, pp. 43-52, 2006.

[15] S. Li, L. Li, and Y. Su, "General iterative methods for a one-parameter nonexpansive semigroup in Hilbert space," Nonlinear Analysis: Theory, Methods E Applications, vol. 70, no. 9, pp. 3065-3071, 2009.

[16] E. Zeidler, Nonlinear Functional Analysis and Its Applications. III: Variational Methods and Optimization, Springer, New York, NY, USA, 1985.

[17] W. Takahashi, Nonlinear Functional Analysis: Fixed Point Theory and Its Applications, Yokohama Publishers, Yokohama, Japan, 2000.

[18] J.-P. Gossez and E. Lami Dozo, "Some geometric properties related to the fixed point theory for nonexpansive mappings," Pacific Journal of Mathematics, vol. 40, pp. 565-573, 1972.

[19] J. S. Jung, "Iterative approaches to common fixed points of nonexpansive mappings in Banach spaces," Journal of Mathematical Analysis and Applications, vol. 302, no. 2, pp. 509-520, 2005.

[20] A. Aleyner and Y. Censor, "Best approximation to common fixed points of a semigroup of nonexpansive operators," Journal of Nonlinear and Convex Analysis, vol. 6, no. 1, pp. 137-151, 2005.

[21] A. Aleyner and S. Reich, "An explicit construction of sunny nonexpansive retractions in Banach spaces," Fixed Point Theory and Applications, no. 3, pp. 295-305, 2005.

[22] R. E. Bruck Jr., "Nonexpansive retracts of Banach spaces," Bulletin of the American Mathematical Society, vol. 76, pp. 384-386, 1970.

[23] S. Reich, "Asymptotic behavior of contractions in Banach spaces," Journal of Mathematical Analysis and Applications, vol. 44, pp. 57-70, 1973. 
[24] K. Aoyama, Y. Kimura, W. Takahashi, and M. Toyoda, "Approximation of common fixed points of a countable family of nonexpansive mappings in a Banach space," Nonlinear Analysis: Theory, Methods \& Applications, vol. 67, no. 8, pp. 2350-2360, 2007.

[25] H. Piri and H. Vaezi, "Strong convergence of a generalized iterative method for semigroups of nonexpansive mappings in Hilbert spaces," Fixed Point Theory and Applications, vol. 2010, Article ID 907275, 16 pages, 2010. 


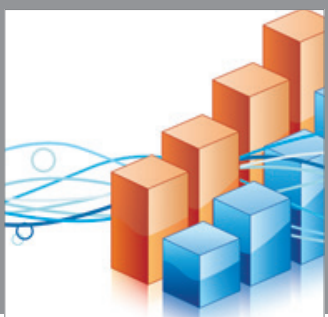

Advances in

Operations Research

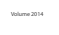

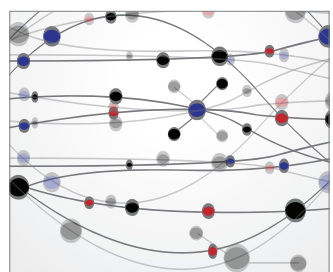

\section{The Scientific} World Journal
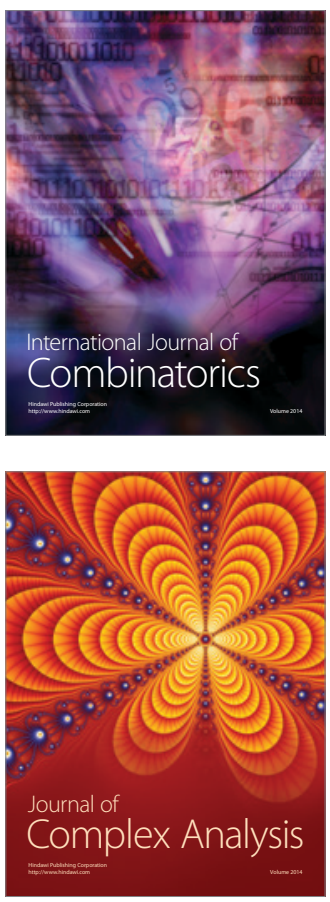

International Journal of

Mathematics and

Mathematical

Sciences
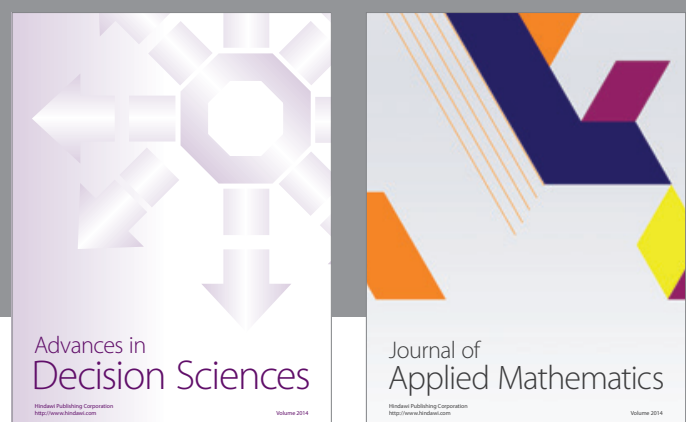

Journal of

Applied Mathematics
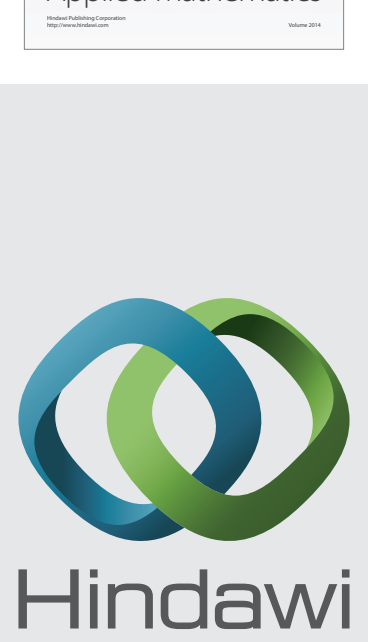

Submit your manuscripts at http://www.hindawi.com
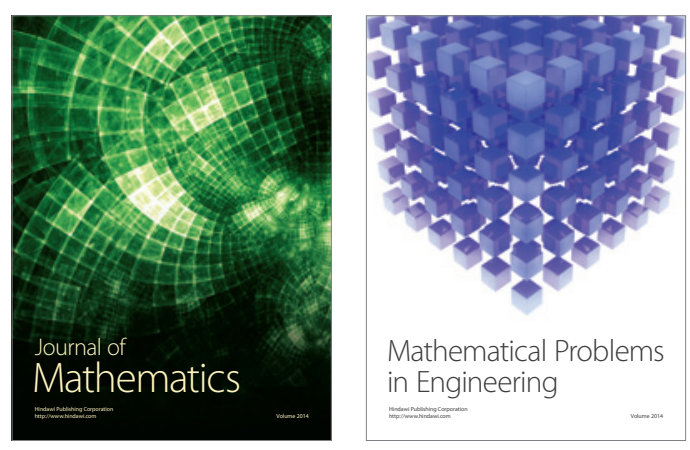

Mathematical Problems in Engineering
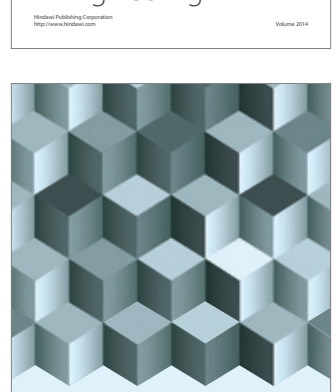

Journal of

Function Spaces
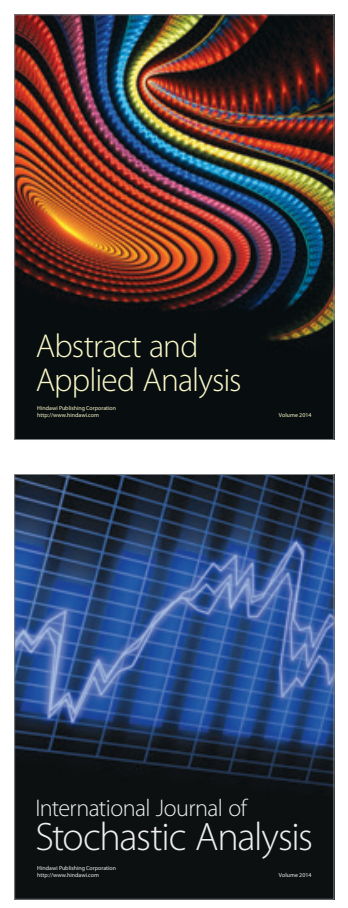

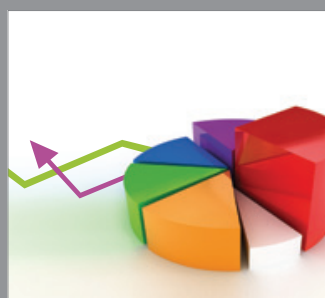

ournal of

Probability and Statistics

Promensencen
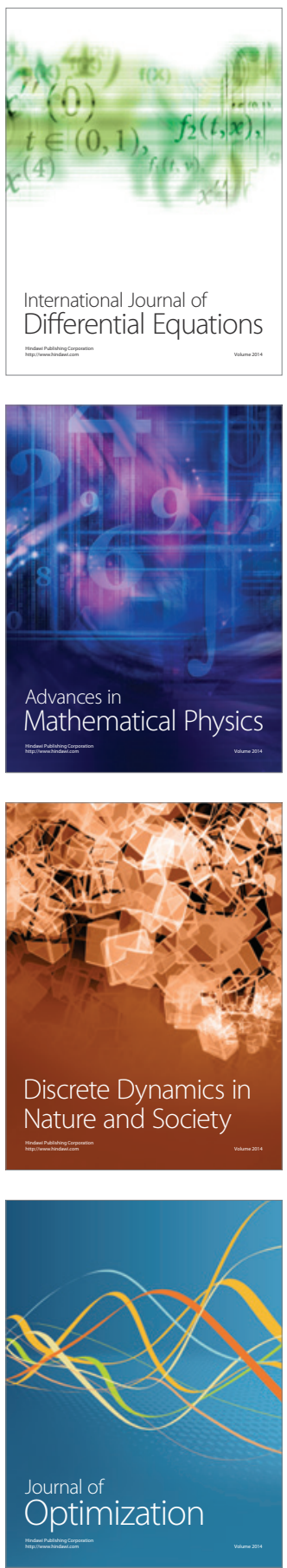